\title{
Escenarios 2020 del Orden Mundial. Análisis desde la Prospectiva Estratégica*
}

\author{
[Versión en español] \\ 2020 World Order Scenarios. Analysis from the Strategic Prospective \\ Cenários da Ordem Mundial 2020. Análise da perspectiva estratégica
}

Recibido noviembre 6 de 2018. Aceptado septiembre 24 de 2019.

Jorge-Isaac Lechuga-Cardozo**
https://orcid.org/0000-0002-0999-5468
México
Oswaldo Leyva-Cordero***
https://orcid.org/0000-0001-9837-020X

México

, Para citar este artículo:

Lechuga-Cardozo, Jorge-Isaac;

Leyva-Cordero, Oswaldo

(2020). Escenarios 2020 del

Orden Mundial. Análisis desde la

Prospectiva Estratégica

Ánfora, 27(48), 141-166. DOI:

https://doi.org/10.30854/anfv27.n48.2020.672

Universidad Autónoma de

Manizales. ISSN 0121-6538/

e-ISSN 2248-6941

\section{Resumen}

Objetivo: el objetivo de este artículo es mostrar evidencia de la prospectiva de los escenarios del orden mundial al año 2020. Metodología: se definió un estudio cualitativo de tipo analítico, no experimental, transeccional y de campo. Se diseñó un instrumento y se aplicó a una muestra de seis expertos en Relaciones Internacionales. Dicho cuestionario presenta cinco ítems para cada variable analizada. Resultados: se logró

\footnotetext{
*Artículo derivado del proyecto de investigación: "Los perfiles exportadores del departamento del Atlántico (Colombia) y el Estado de Nuevo León (México): análisis comparativo", financiado por la Universidad Autónoma de Nuevo León, iniciado el 22 de enero de 2018.

** Magíster en Administración. Administrador de Empresas. Doctorando en Relaciones Internacionales, Negocios y Diplomacia. Docente de la Universidad Autónoma de Nuevo León. Correo: jorge.lechugacrd@ uanl.edu.mx

*** Doctor en Gerencia y Política Educativa. Maestro en Políticas Públicas. Ingeniero en Electrónica y Comunicaciones. Docente Titular-Investigador de la Universidad Autónoma de Nuevo León, México. Correo: oswaldo.leyva@uanl.mx
} 
la clasificación de las variables multipolaridad, liderazgo Asia Pacífico, unipolaridad, amenaza a la seguridad mundial y neoproteccionismos; estas fueron catalogadas según la metodología de Swartch en cinco escenarios del orden global a 2020. Conclusiones: se concluye que la misma magnitud y velocidad de cambio resultantes de la globalización permitirán en la arena global el surgimiento de nuevos poderes, nuevos desafíos de gobernabilidad y una sensación más generalizada de inseguridad, que incluye el terrorismo.

Palabras-clave: Orden mundial; Globalización; Escenarios de Prospectiva; Poder político.

\section{Abstract}

Objective: the objective of this article is to show evidence of the forecast of the world order scenarios for the year 2020. Methodology: defined as a qualitative, analytical, non-experimental, transectional and field type study. An instrument was designed and applied to a sample of six International Relations experts. Said instrument is a questionnaire consisting of five entries for each of the analyzed variables. Results: a classification of the following variables: multipolarity, Asia Pacific leadership, unipolarity, threat to world security and neoprotectionism was achieved. These were classified according to the Swartch methodology in five scenarios of the global order for 2020. Conclusions: the conclusion shows that the same magnitude and speed of change emerging from globalization would allow, in the global arena, the emergence of new powers, new governance challenges, and a more widespread sense of insecurity, terrorism included. 
Keywords: World order; Globalization; Prospective Scenarios; Political Power.

\section{Resumo}

Objetivos: o objetivo deste artigo é mostrar evidências dos cenários mundiais em perspectiva para o ano 2020. Metodologia: foi definido um estudo qualitativo do tipo analítico, não experimental, transecional e de campo. Um instrumento foi projetado e aplicado a uma amostra de seis especialistas em Relações Internacionais. Este questionário apresenta cinco itens para cada variável analisada. Resultados: a classificação das variáveis multipolaridade, liderança na Ásia-Pacífico, unipolaridade, ameaça à segurança mundial e neoprotecionismo foi alcançada; estas foram catalogadas de acordo com a metodologia Swartch em cinco cenários da ordem global até 2020. Conclusões: conclui-se que a mesma magnitude e velocidade de mudança resultantes da globalização permitirão na arena global o surgimento de novos poderes, novos desafios de governança e um sentimento mais amplo de insegurança, que inclui o terrorismo.

Palavras-chave: Ordem mundial; Globalização; Cenários prospectivos; Poder político. 


\section{Introducción}

El mundo transita a un reequilibrio luego de la posguerra fría, a través de nuevas formas de conexión y acción con las que occidente pierde poder; es decir, está frente a una contrarrevolución geopolítica. La revolución geopolítica del siglo XVIII ha empezado a tener una contrarrevolución que se presenta desde finales del siglo XX y que ha marcado la ruta del siglo XXI: el resurgimiento del viejo mundo no occidental que se expresa como el restablecimiento del equilibrio fracturado entre grandes Estados, imperios e incluso civilizaciones (Patiño, 2017).

$\mathrm{Al}$ respecto, Ohmae (2005) postula que el nuevo escenario global requiere de una visión del mundo en la que la apertura, la flexibilidad, la interacción, los nuevos sistemas educativos, el comercio global, adquieren cada vez mayor relevancia, junto al surgimiento del Estado Región, como protagonista del desarrollo. Precisamente, en estos nuevos contextos y tendencias globales, como apunta Barbieri (1993), el futuro es una necesidad, así como una elección, que permite las posibles consecuencias de las acciones de hoy con la intención de construir un futuro deseado y, finalmente, es una actitud para concebir la vida, el mundo y los comportamientos individuales y sociales.

Ahora bien, el presente artículo recoge el diseño de cinco escenarios posibles del orden mundial a 2020, a través de la entrevista a expertos. Con la aceleración del cambio, la subida de las interdependencias y de las incertidumbres, se impuso cada vez más la necesidad de tener en cuenta una multitud de escenarios y, en cada caso examinar, cual estrategia óptima podría ser adoptada.

Cabe desatacar que este estudio goza de utilidad en la medida en que es un ejercicio de prospectiva crítica frente a los escenarios de futuro del orden mundial, cuya perspectiva mitiga la incertidumbre, el riesgo y la complejidad que caracteriza el siglo XXI. La disciplina de las relaciones internacionales exige el análisis de los procesos y fenómenos mundiales a partir de su evolución futura en el corto, mediano y largo plazo (Batta, 2013, p. 44); así, la técnica de los escenarios de Schwartz (1997) cobra importancia, ya que permite una proyección científica de los escenarios del orden mundial. Agrega González (2015) que "la prospectiva como recurso metodológico se convierte en una herramienta de análisis para la determinación de escenarios en el ámbito social, particularmente en las relaciones internacionales" (p. 87).

En este sentido, Schwartz (1997) subraya la necesidad de entrenar los contemporáneos para que enfoquen futuros posibles de manera que no se encuentren desarmados cuando de pronto se transforme la escena sobre la cual ellos 
operan; este cambio exige de su parte la adopción de una estrategia distinta de la que se ha utilizado antes (Schwartz, 1997, p. 201).

De acuerdo con Schwartz, los escenarios son un medio de articular y ordenar la gama de incertidumbres esenciales, susceptibles de afectar los resultados de los planes en curso. La calidad de un escenario no se mide por la capacidad de hacer predicciones correctas, pero sí por la manera en la cual sabrán estimular la intuición, ayudar a comprender y conducir a una acción eficaz. Los escenarios son historias que tienen por tema el futuro y son, entonces, perfectamente posibles de suceder o no, puesto que ellos tienen un impacto psicológico que no lo tienen los diagramas y las ecuaciones (Schwartz, 1997, p. 203).

En cuanto a la estructura del artículo se presentará de la siguiente manera: luego del marco teórico, se expondrá la metodología utilizada para la presente propuesta. Después, se realizará la presentación de los cinco escenarios, a partir análisis de la información recolectada. Para finalmente llegar al planteamiento de la discusión y conclusiones.

\section{Marco teórico}

A continuación, se definen las variables multipolaridad, liderazgo Asia Pacífico, unipolaridad, amenaza a la seguridad mundial y neoproteccionismos, a partir de la revisión de los principales autores en el tema.

\section{Aproximación al diseño de Escenarios Prospectivos}

Figura 1. Concepción del futuro como una realidad múltiple

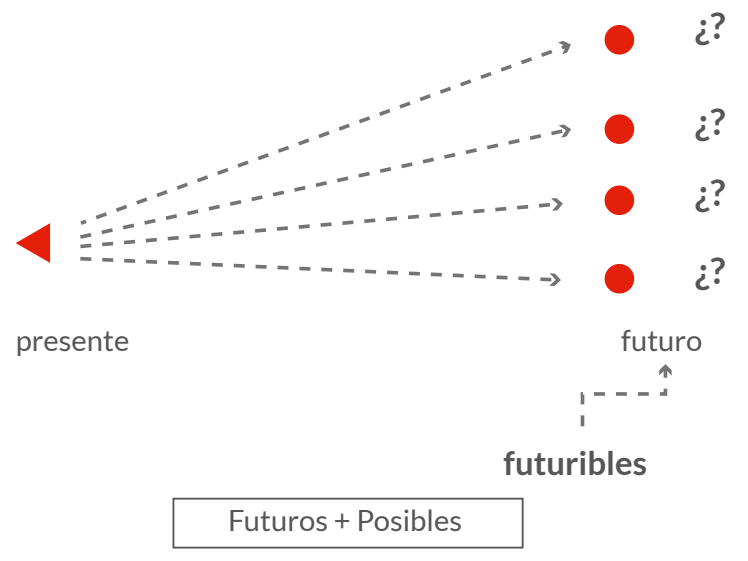

Fuente: Mojica, (2010, p. 9) 
En principio, Bertrand de Jouvenel en su obra El Arte de la Conjetura, demuestra que no existe un solo futuro sino muchos futuros posibles a los que él llama "futuribles". Se refiere a que si el futuro es múltiple y no único desaparece el interés por predecirlo, de modo que los diferentes futuros posibles tienen solamente la función de mostrar alternativas de lo que podría suceder. Estas alternativas se denominan "escenarios", los cuales cumplen solamente la función de señalar situaciones eventuales que podrían acaecer años adelante.

Figura 2. Elección de un futuro entre varios "futuros posibles"

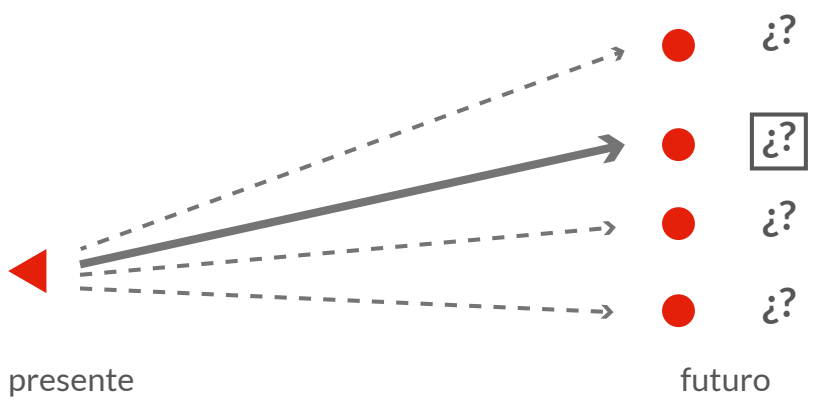

Fuente: Mojica (2010, p. 10)

Ahora, lo más importante del futuro como realidad múltiple radica, por consiguiente, en poder comparar los diferentes futuros, examinar las implicaciones que tendría cada uno si llegase a suceder y, finalmente, elegir el más conveniente. En el diseño de escenarios futuros converge toda la información que se ha recabado hasta el momento actual, en particular, las ideas que provienen de las tendencias y mejores prácticas mundiales. El diseño del futuro se logra necesariamente a través de la redacción de escenarios. Y entre las herramientas más aconsejables está la cruz de escenarios de Schwartz (1997), que es muy simple porque reduce las alternativas de escenarios solamente a cuatro (Mojica, 2010, p. 41; Lechuga et al., 2018, p. 133). A partir de los escenarios de Schwartz se diseña un escenario apuesta, tres escenarios alternativos y un escenario catastrófico, como se observa a continuación. 


\section{Multipolaridad del Orden Mundial.}

De acuerdo con Trejos (1997), el escenario probable será el de una interdependencia de los países casi absoluta. Apunta Trejos que la globalización económica está consolidada y las fronteras nacionales serán apenas linderos demarcadores de países inter-penetrados (p. 89) Añade que la integración económica ha tejido lazos entre las naciones que las volvió interdependientes; de este modo, la conformación de bloques comerciales ha facilitado acuerdos multilaterales que se tornan hegemónicos en la conducción económica mundial.

Paralelamente, los tradicionales centros de decisión localizados en occidente están siendo reemplazados en importancia internacional por nuevos centros de decisión en países emergentes, de los que depende la economía mundial; así, el océano Pacífico será el centro de gravedad estratégico mundial (Pérez, 2012; Schiavon et al, 2014; Zeraoui et al., 2014; Pearson y Rochester, 2000).

De igual forma, Pérez (2012) dice que las potencias occidentales se han enriquecido desarrollando la terciarización de sus economías y apostando a campos como la tecnología, la innovación, la investigación, etc. Por otro lado, los tradicionales sectores primario y secundario, dedicados a la extracción y a la fabricación, se han trasladado a países del sudeste asiático, oriente medio o Latinoamérica; esto, ha permitido que nuevas economías se desarrollen como productoras de materias primas y se hayan especializado en la fabricación y confección.

Ahora bien, los procesos de deslocalización y las inversiones internacionales directas que desde las potencias tradicionales han tenido lugar hacia otras zonas del planeta han permitido que surjan nuevos países etiquetados como emergentes, que están encaminados a liderar la economía en un futuro no muy lejano. En este orden de ideas, Manrique (2016) apunta que los países en desarrollo deben iniciar de manera urgente el nuevo camino hacia modelos de crecimiento que mejoren su productividad y competitividad, dejando de lado la dependencia hacia los commodities, de tal modo que se dé vuelta a su desaceleración.

\section{Poderio Económico, Político y Social de Asia Pacífico.}

Todo parece indicar que el siglo XXI estará dominado por las economías asiáticas; la zona del atlántico norte ira perdiendo poder relativo en favor de Asia- Pacífico. En este sentido, se observa cómo China se está convirtiendo en un socio comercial estratégico de países como Alemania. El auge de Asia está llamado a continuar en próximos decenios.

Por su parte, en el Mapping the Global Future (National Intelligence Council, 2004) se presenta el escenario del mundo post-Davos, en el que ilustra 
cómo un fuerte crecimiento económico, liderado por la China y la India, puede remodelar el proceso de globalización dándole un rostro menos occidental, trasformando el campo de juego político, pero manteniendo las desigualdades sociales. No sería posible en este contexto el establecimiento de un Fondo Monetario Asiático y, menos probable, una Organización Asiática del Comercio.

Según un estudio prospectivo reciente del Banco Asiático de Desarrollo, BAsD (2011), la parte de Asia en el producto bruto mundial pasará del 26\% en 2010 al 51\% en 2050, convirtiendo plenamente al siglo XXI en el "siglo de Asia". Al respecto, Bustelo (2011) agrega que

Asia-Pacífico, con la excepción de Japón en algunos campos, ha destacado durante los últimos decenios por su alto crecimiento económico, su creciente peso en el comercio internacional, el incremento de su consumo de energía y de otras materias primas, sus crecientes inversiones extranjeras y en el extranjero y su cada vez mayor potencial financiero, medido por el incremento de sus reservas en divisas (p. 11).

En este orden de ideas The Asian Development Bank, ADB (2011) sustenta que Asia está en medio de una transformación histórica. Si continúa su trayectoria reciente, en 2050 su ingreso per cápita podría aumentar seis veces en términos de paridad de poder adquisitivo (PPA) hasta alcanzar los niveles de Europa; ello, haría que unos 3.000 millones de asiáticos adicionales fueran ricos gracias a la corriente estándares. Casi doblando su participación en el producto interno bruto (PIB) mundial a 52\% en 2050, Asia recuperaría la posición económica dominante que tenía hace unos 300 años, antes de la revolución industrial y China mantendrá la capacidad de seguir siendo competitiva aún en un escenario de precio del petróleo bajo (Manrique, 2016).

\section{Unipolaridad: Hegemonía Estadunidense.}

La configuración actual del mundo se basa en el predominio de Estados Unidos, principal potencia política, económica, cultural y militar. De esta forma, las decisiones mundiales vienen determinadas e influenciadas por EE. UU. y sus principales socios: Europa y Japón (Pérez, 2012). En Mapping the Global Future de la National Intelligence Council (2004) se presenta el escenario Pax americana, en el que el que se postula que el predominio de los Estados Unidos sobrevive a los cambios radicales en el panorama político global y permite dar paso a un nuevo orden global e incluyente haciendo frente a las demandas de energía y al agotamiento de la unipolaridad estadounidense (National Intelligence Council, 
2004). En este sentido, Estados Unidos seguirá siendo el actor más poderoso en lo económico, tecnológico y militar.

\section{Amenaza a la Seguridad Global.}

El islam político sigue siendo una fuerza potente: de ahí que se proyecte que se fortalezcan los movimientos globales alimentados por políticas identitarias de radicalismo religioso, convirtiéndose en una amenaza a las normas y valores mundiales. De acuerdo con lo anunciado en Mapping the Global Future (National Intelligence Council, 2004) en su escenario "Ciclo del miedo", crece la preocupación por el terrorismo internacional y los conflictos internos hasta tomar medidas de seguridad extremas para prevenir ataques mortales y hasta producir un mundo orwelliano con nuevos centros de poder mundial.

De modo similar, Davis (2015) lo prospecta como "Mad Max", un mundo caracterizado por conflictos internacionales y nacionales, donde los gobiernos ejercen fuertes controles de seguridad tanto en los negocios como en la sociedad. Los niveles de financiación para los desafíos sociales y de desarrollo serán limitados. Todo esto alimentado por factores como el fortalecimiento de redes terroristas más diversificadas que produzcan una alianza antioccidental.

\section{Recesión Económica Global y Surgimiento de Políticas Neoproteccionistas.}

La Cumbre del G20 que se celebró en la localidad china de Hangzhou tuvo como eje central de sus debates la reactivación del comercio mundial, pues la economía global estaría amenazada por el riesgo de retorno a escenarios de recesión agravado por la aparición de medidas neoproteccionistas en los países del Primer Mundo, especialmente tras la llegada de Donald Trump al poder en EE. UU. (Gorraiz, 2016).

Así pues, China estaría inmersa en una crisis económica debido a su limitada integración financiera internacional, su aislamiento y control del aparato estatal interno e ineficiente asignación de recursos económicos. Por su parte, la contracción mundial en los commodities estaría afectando las exportaciones de América Latina y la depreciación de sus monedas, debido a la apreciación del dólar que se traduce en aumentos en los costes de producción, tasas de inflación y deuda externa, así como pérdida de competitividad.

En palabras de Lagarde (citada por Gorraiz, 2016), directora del FMI "la fortaleza del dólar junto con la debilidad de los precios de los productos crea 
riesgos para los balances y financiación de los países deudores en dólares” (p. 2 lo que podría estar causando aumento de la inestabilidad social, tasas de pobreza y retroceso de las libertades democráticas. El estancamiento en la economía mundial se puede dar si algunos países en vía de desarrollo entran en crisis después del desplome de los commodities, causando un efecto dominó que puede llegar a la declaración de quiebra de muchas empresas.

Agrega Manrique (2016) que las previsiones de desaceleración económica indican una actividad económica mundial casi plana y el desequilibro entre la oferta y demanda continuará, por lo que se prevé un ciclo largo con precio del petróleo bajo (p.1).

Por otro lado, la salida de EE. UU. de la Asociación Transpacífico (TPP) y la actual renegociación entre EE.UU., Canadá y México del NAFTA o TLCAN, aunado al tratado Transatlántico de Comercio e Inversión que, a pesar de que busca la eliminación de barreras arancelarias, prevé barreras administrativas como las normas alimentarias y fitosanitarias. Esto lleva a pensar en el fin de los tratados comerciales trasnacionales (TTIP, NAFTA y TTP) y a la implementación por las economías del primer mundo de medidas proteccionistas frente a los países emergentes, cuyo paradigma sería el establecimiento de medidas antidumping contra el acero chino con aranceles que oscilarán del 20\% europeo al 500\% estadounidense y que significarán el retorno al neoproteccionismo económico.

\section{Metodología}

La investigación de la que se origina este artículo se realizó desde un enfoque cualitativo, con una metodología cualitativa de tipo analítica y un diseño no experimental, transeccional y de campo. Las variables trabajadas (multipolaridad, liderazgo de países asiáticos, unipolaridad, amenaza a la seguridad mundial, neoproteccionismos) se analizaron a través de expertos en relaciones internacionales en la ciudad de Monterrey (México).

En este caso, el enfoque investigativo de la presente investigación es cualitativo, definido por Hernández, Batista y Fernández (2014) como el que "utiliza la recolección y análisis de los datos para afinar las preguntas de investigación o revelar nuevas interrogantes en el proceso de interpretación” (p. 7). Y agregan Taylor y Bogdán (1986) que la investigación cualitativa "es aquella que produce datos descriptivos: las propias palabras de las personas, habladas o escritas, y la conducta observable" (p. 11).

En este sentido, autores como Mendoza (2006) destacan la importancia de la investigación cualitativa en la medida en que permite descubrir y refinar las 
preguntas de investigación que guiarán a los instrumentos utilizados para la obtención de la información. Así como Rodríguez y Gorjón (2014) resaltan que este tipo de estudios ofrecen un sentido profundo de conocimiento acerca de las actitudes, comportamiento, percepciones y preferencias de las personas.

Así pues, el alcance establecido es el analítico o interpretativo que pretende encontrar pautas de relación internas en un evento para llegar a un conocimiento más profundo de éste, que la mera descripción. Para ello, se vale de las matrices de análisis, que proporcionan los criterios que permiten identificar esas pautas de relación. La investigación analítica intenta desentrañar lo que está más allá de lo evidente (Hurtado, 2010, p. 133).

A este propósito, Hernández, Fernández y Baptista (2014) afirman que la investigación interpretativa intenta encontrar sentido a los fenómenos en función de los significados que las personas les otorguen (p. 9). Además, permite alcanzar un diseño...no experimental "Que se realiza sin la manipulación deliberada de variables y en los que sólo Se observan los fenómenos en su ambiente natural para después analizarlos" (Hernández et al., 2014, p. 149). Y de tipo transversal, porque el estudio que se busca hacer no tendrá un seguimiento en el tiempo y se efectuará en un período determinado (Hernández et al., 2014, p. 154).

\section{Participantes}

Para el presente trabajo se seleccionaron 8 expertos en relaciones internacionales de Monterrey. El tipo de muestreo es "no probabilístico", es decir, la muestra se seleccionó de acuerdo con la disposición de atención al libreto de entrevista en el campo. La selección de expertos se realiza con base en las siguientes características: Disponibilidad y accesibilidad para contestar la entrevista, conocimiento técnico del fenómeno orden global, ubicación geográfica en Nuevo León y formación académica mínima de Maestría.

Cabe resaltar que la utilización de n cantidad de expertos es válida en la medida en que una muestra cualitativa busca significados (Martínez-Salgado, 2012), que permitan lo que Sandelowski y Barroso (2003) llaman generalización ideográfica. El criterio más generalmente compartido en metodología cualitativa es el de la saturación, que tiene lo que Carlsen y Glenton (2011) llaman un rol dominante, aunque difícil de operativizar. La propuesta consiste en que dejamos de recoger unidades muestrales en el trabajo de campo cuando las nuevas unidades seleccionadas dejan de aportar nueva información relevante con respecto a las anteriores (García-Hernández y Manzano-Caudillo, 2010).

Por otro lado, agregan Creswell y Miller (2000) que en la investigación cualitativa los resultados son precisos desde el punto de vista del investigador, el 
participante o los lectores de un informe de investigación; en palabras de Strauss y Corbin (1990) en la teoría fundamentada se da la validez suplementaria al relacionar datos con categorías y contrastar los datos con la literatura. De modo que la validez interna está dada por los autores y la validez externa por la calidad del experto.

Tabla 1. Expertos a los que se les aplicó la entrevista

\begin{tabular}{|c|c|c|}
\hline Nombre & Puesto & Institución \\
\hline $\begin{array}{c}\text { Mtro. Flavio Rafael González } \\
\text { Ayala }\end{array}$ & $\begin{array}{l}\text { Investigador en Estudios de } \\
\text { Asia y África }\end{array}$ & $\begin{array}{c}\text { Universidad Autónoma de } \\
\text { San Luis Potosí }\end{array}$ \\
\hline $\begin{array}{c}\text { Mtro. Joel Angel Bravo An- } \\
\text { duaga }\end{array}$ & Investigador en Geopolítica & $\begin{array}{c}\text { Instituto Tecnológico de } \\
\text { Estudios Superiores de } \\
\text { Monterrey - Campus } \\
\text { Querétaro }\end{array}$ \\
\hline $\begin{array}{c}\text { Mtra. Karen Jeraldín Ramos } \\
\text { Lizárraga }\end{array}$ & $\begin{array}{c}\text { Coordinadora Academia de } \\
\text { Turismo Facultad de Cien- } \\
\text { cias Políticas y Relaciones } \\
\text { Internacionales }\end{array}$ & $\begin{array}{l}\text { Universidad Autónoma de } \\
\text { Nuevo León }\end{array}$ \\
\hline Mtro. Antonio Galván & $\begin{array}{l}\text { Gerente de Ventas de } \\
\text { Exportación }\end{array}$ & Trophe \\
\hline Mtro. Carmelo Santillán Ramos & CEO & CSR Consulting \\
\hline Mtra. Sandy Martínez Lara & $\begin{array}{c}\text { Investigadora en } \\
\text { Relaciones Internacionales }\end{array}$ & $\begin{array}{c}\text { Universidad Autónoma de } \\
\text { Nuevo León }\end{array}$ \\
\hline Mtra. Priscila Vera Zamora & $\begin{array}{c}\text { Investigadora en Política } \\
\text { Exterior }\end{array}$ & $\begin{array}{c}\text { Universidad Autónoma de } \\
\text { Nuevo León }\end{array}$ \\
\hline $\begin{array}{l}\text { Mtro. Alejandro Castillo } \\
\text { Maldonado }\end{array}$ & CEO & $\begin{array}{c}\text { Camu Worldwide Hong } \\
\text { Kong Ltd. }\end{array}$ \\
\hline
\end{tabular}

Fuente: elaboración propia.

Tabla 2. Ficha técnica de la investigación

\begin{tabular}{c|c}
\hline Universo & $\begin{array}{c}\text { Expertos en Relaciones Internacionales en } \\
\text { Monterrey (México) }\end{array}$ \\
\hline Ámbito & Regional, Monterrey (México) \\
\hline Método de recogida de información & $\begin{array}{l}\text { Entrevista semi- estructurada, } \\
\text { suministrada presencialmente }\end{array}$ \\
\hline
\end{tabular}




\begin{tabular}{c|c}
\hline Unidad muestral & $\begin{array}{c}\text { Expertos en Relaciones Internacionales en } \\
\text { Monterrey (México) }\end{array}$ \\
\hline Tamaño de la muestra & 9 \\
\hline Trabajo de campo & Octubre de 2018 \\
\hline Número de entrevistas realizadas & 9 \\
\hline Respuestas válidas & 9
\end{tabular}

Fuente: elaboración propia.

\section{Instrumentos}

Se diseñó un instrumento de conformidad con las cinco variables (multipolaridad, liderazgo Asia Pacífico, unipolaridad, amenaza a la seguridad mundial, neoproteccionismos), el cual se aplicó a 8 expertos en relaciones internacionales en Monterrey. Dicho instrumento contempla 5 ítems con respuestas abiertas y se construyó con base en las dimensiones evaluadas en la investigación. Así, la técnica utilizada, la entrevista, estuvo orientada a establecer contacto directo con las personas que se consideraron fuente de información adecuada para este estudio, si bien puede soportarse en un cuestionario flexible, su propósito es obtener información espontánea y abierta (Bernal, 2010, p. 177).

Tabla 3. Estructura del guion de la entrevista

\begin{tabular}{c|c|c|} 
Categorías de Análisis & Número de preguntas & Autores \\
\hline Multipolaridad & 1 & $\begin{array}{c}\text { Trejos (1997), Pérez } \\
\text { (2012), Manrique (2016) }\end{array}$ \\
\hline Liderazgo Asia Pacífico & 1 & $\begin{array}{c}\text { National Intelligence Coun- } \\
\text { cil (2004), BAsD (2011), } \\
\text { Bustelo (2011), Manrique } \\
\text { (2016) }\end{array}$ \\
\hline Unipolaridad & 1 & $\begin{array}{c}\text { Pérez (2012), National } \\
\text { Intelligence Council (2004) }\end{array}$ \\
\hline Amenaza a la seguridad mun- \\
dial & 1 & $\begin{array}{c}\text { National Intelligence Coun- } \\
\text { cil (2004), Davis (2015) }\end{array}$ \\
\hline Neoproteccionismos & 1 & $\begin{array}{c}\text { Gorraiz (2016), Manrique } \\
\text { (2016) }\end{array}$ \\
\hline
\end{tabular}

Fuente: elaboración propia. 


\section{Procedimiento}

Inicialmente se aplicó un guion de entrevista a una selección de expertos en relaciones internacionales; a partir de allí, se recopiló la información a través del software Nvivo, que permitió la ilustración de la frecuencia de las respuestas de los expertos en gráficas de nube para cada uno de los 5 escenarios propuestos por la metodología Schwartz (1997); seguidamente, se elaboraron las conclusiones y recomendaciones a la luz del objetivo de investigación (Bascón et al., 2016, p. 39; Lechuga, 2018, p. 196).

\section{Resultados}

Las variables multipolaridad, liderazgo Asia Pacífico, unipolaridad, amenaza a la seguridad mundial y neoproteccionismos, fueron clasificadas según la metodología de Swartch en escenarios, como se acompañan en los subtítulos a continuación.

\section{Multipolaridad: Escenario Apuesta}

Como parte de la revisión de datos, se procedió a identificar cuáles eran las palabras más frecuentes utilizando el programa Nvivo 11 sobre un escenario multipolar donde la palabra que asociaban con mayor periodicidad en las entrevistas es países, además de las palabras mundo, emergentes, multipolar y económico (Figura 3). Resalta que la configuración actual del mundo se basa en el predominio de Estados Unidos y sus socios Europa y Japón y que el surgimiento de nuevos núcleos de poder sería una oportunidad para la participación de más países en la toma de decisiones al incluirse nuevos puntos de vista en las mesas de diálogo internacional.

Este cambio en el orden mundial, basado en un rebalanceo de la economía hacia los países emergentes, permitirá mayor pluralidad en la toma de decisiones en asuntos globales. El rápido crecimiento de los países emergentes los llevará a demandar mayores cuotas de poder en las instituciones internacionales. Los problemas de endeudamiento y desempleo de los países avanzados podrían dar un auge a las presiones proteccionistas, lo que podría generar conflictos; los países emergentes están dispuestos a quitarle el liderazgo mundial a Estados Unidos y Europa y hacia el año 2020 los esfuerzos de dichos países se continúan afianzando, como es el caso de los BRICS, de los cuales China e India se perfilan como potencias para el 2050 . 
Figura 3. Escenario multipolar

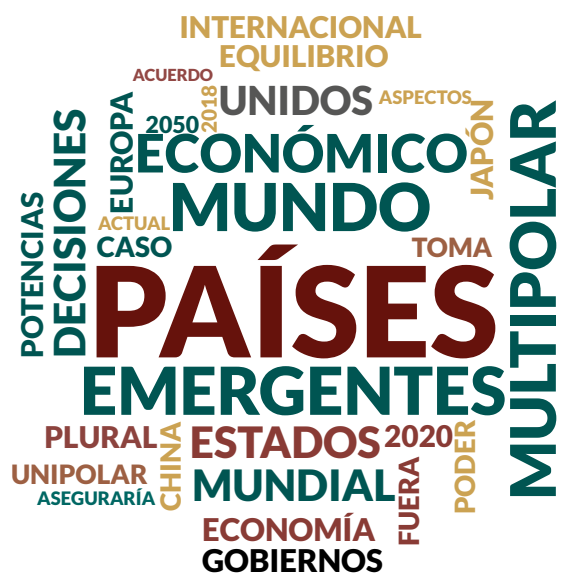

Fuente: elaboración propia a través de en Nvivo

Según los expertos existen poderes revisionistas en la arena mundial que cuestionan el statu quo en materia política, económica y militar del mismo establecimiento, como son China, Rusia e Irán, las cuales ejercen poder de influencia en sus respectivas áreas geográficas (Rodríguez, 2017). El objetivo de los países emergentes y del Tercer Mundo es diversificar los centros de poder político y económico para tener voz en las decisiones de carácter global. Se espera que las decisiones internacionales estén condicionadas no solo por potencias mundiales sino también regionales (México, Indonesia, Nigeria). Este escenario multipolar permitirá la participación de más países en la agenda global y la definición de nuevos rumbos al incluirse mayor cantidad de ópticas en el diálogo internacional. 


\section{Liderazgo de Asia Pacífico. Escenario Alternativo 1}

Figura 4. Escenario Liderazgo de Asia Pacifico

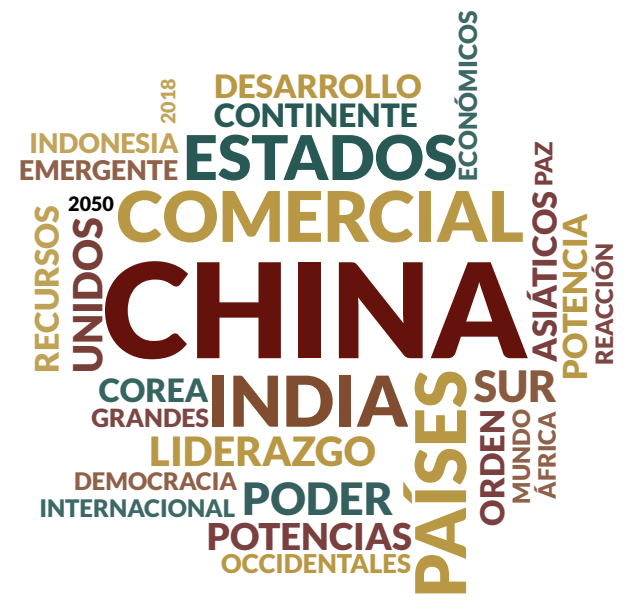

Fuente: elaboración propia a través de Nvivo.

La percepción de los expertos mostró las siguientes palabras: China, India, comercial, países y liderazgo. El orden global tiende hacia el liderazgo de China, y en ese continente se encuentran los posibles países líderes en el mundo, de acuerdo con Funabashi, Oksenberg y Weiss (1994) allí se encuentran India, Corea del Sur y obviamente Japón. China es una potencia emergente, cuyo fundamento esencial de no alterar el orden internacional con demasiado ímpetu, sino empleando instrumentos económicos para alcanzar el estatus de potencia mundial. A fin de evitar críticas a sus ambiciones hegemónicas, los funcionarios chinos llaman a esta estrategia "desarrollo pacífico".

Otros han alegado que "la reacción de China ante el predominio de Estados Unidos implica una combinación de acuerdos, competencia y resistencia de baja intensidad” (Paul, 2011). Esto se explica, porque a pesar de ser una potencia emergente China “es aun sustancialmente más débil que Estados Unidos” (Paul, 2011). Sin embargo, China parece buscar un equilibrio duro con Asia Meridional; en un esfuerzo por equilibrar el poder de India ha ampliado su vieja relación estratégica con Pakistán. Este tipo de respuesta al ascenso de China se explica también por el hecho de que ese país ha adoptado dos de los elementos esenciales de la paz liberal, a saber: la interdependencia económica y las instituciones internacionales, pero ha rechazado el tercero, la democracia. El déficit de democracia en China genera una gran incertidumbre para sus vecinos asiáticos y para Estados Unidos, porque sus intenciones no son fáciles de descifrar (Figura 4). 


\section{Unipolaridad. Escenario Alternativo 2}

Figura 5. Escenario Unipolar

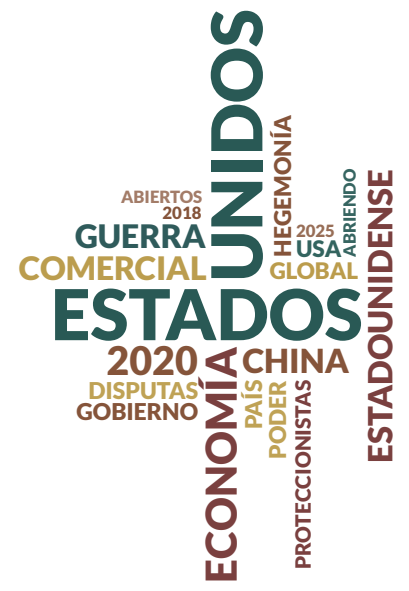

Fuente: elaboración propia a través de Nvivo

La percepción de los expertos mostró las siguientes palabras: Estados Unidos, economía, comercial, China y disputas. En este sentido, los expertos expresan que la hegemonía se ha perdido con el paso de los años; cuando terminó la guerra fría y se disolvió la URSS, EE. UU. parecía ser el hegemon, pero la misma libertad de recursos con los que predicaba el capitalismo, doctrina apoyada por EE. UU, llevó a que el mundo no se pudiese desarrollar en un ámbito unipolar, sino que se fuese abriendo a opciones emergentes y diferentes economías hacia la multipolaridad. Así, EE. UU seguirá buscando preservar su supremacía e institucionalizar la unipolaridad como paradigma de las relaciones internacionales orientada a posicionarlo como una superpotencia que no admitirá cuestionamientos. No hay lugar donde esto se exprese en forma más clara que en Medio Oriente, donde los equilibrios políticos y geopolíticos fueron fuertemente sacudidos por la guerra de ocupación y sus efectos recientes (Figura 5). 


\section{Amenaza a la Seguridad Mundial. Escenario Alternativo 3}

Figura 6. Escenario Amenaza a la Seguridad

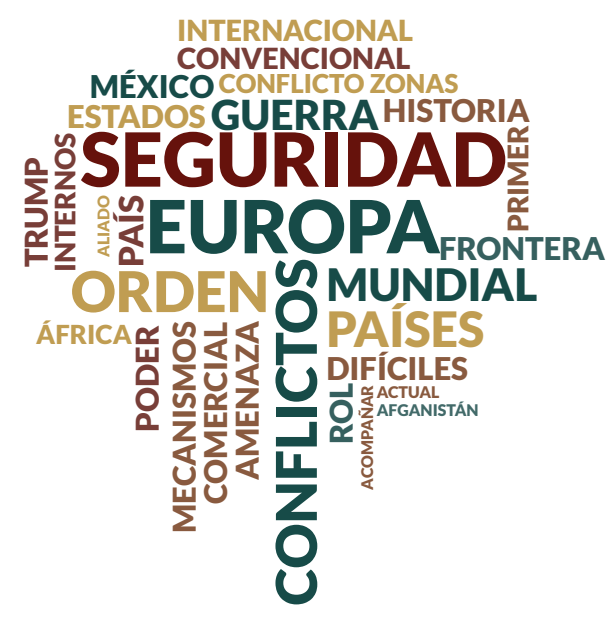

Fuente: elaboración propia a través de Nvivo

La percepción de los expertos mostró las siguientes palabras: conflictos, seguridad, orden mundial, Europa y guerra. Al referirse a la amenaza a la seguridad destacan que Europa carece de un poderío militar de primer orden. Su nueva política común de defensa y seguridad está meramente encaminada a apoyar a la ONU y OTAN en el rescate y estabilización de Estados fallidos.

Europa, además está viviendo momentos difíciles en materia migratoria, ya que enfrenta una oleada sin precedentes en la historia reciente de inmigrantes que huyen literalmente del horror de las guerras en sus países de origen (Siria, Afganistán, Norte de África y el África Subsahariana). Por todo ello, Europa se verá forzada a formar un área de libre comercio con los países mediterráneos que atenúe las presiones sobre sus fronteras. Por todas estas consideraciones, parece que el rol de Europa en la lucha por un nuevo orden internacional tendrá que limitarse básicamente a acompañar a EUA, aportando mercados, tecnología, tradiciones culturales y otras variedades de poder blando (Figura 6). 


\section{Neoproteccionismo l. Escenario Catastrófico}

Figura 7. Escenario Neoproteccionista

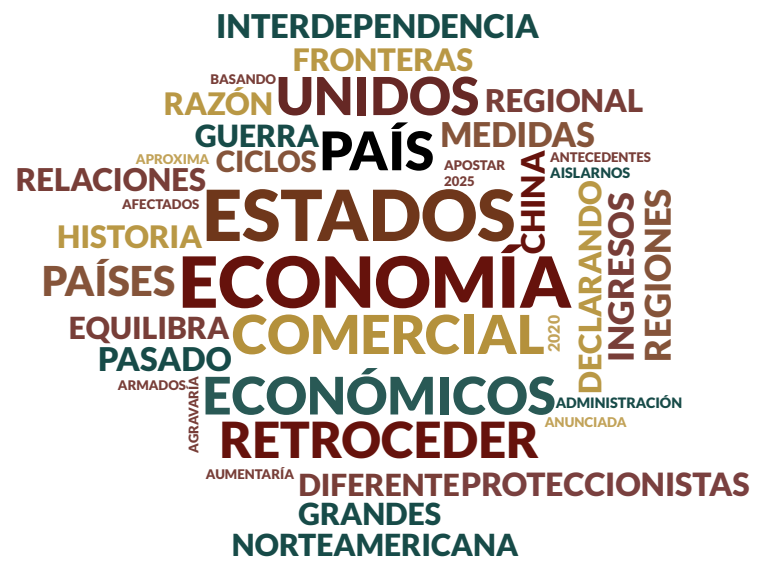

Fuente: elaboración propia a través de Nvivo

La percepción de los expertos mostró las siguientes palabras: Estados Unidos, país, economía, comercial, y económicos. Se hace mención a que la recesión ya ha sido anunciada por el FMI y se aproxima; no obstante, la economía estadounidense mantiene indicadores económicos positivos hasta la fecha; sin embargo, algunos expertos económicos vaticinan una tormenta perfecta y el proteccionismo resurge en la zona de influencia norteamericana.

Ahora bien, la última administración estadounidense está empeñada en deshacer varios tratados importantes que generan grandes ingresos económicos al país, al igual que equilibra la economía regional. Debido a las medidas proteccionistas, Estados Unidos podría retroceder encerrándose en sus propias fronteras y declarando una guerra comercial con China, que es una historia completamente diferente; es un país que está centrado en concretar sus metas hacia el año 2025, basando su economía planificada 4.0 que incluye el desarrollo tecnológico en sectores clave y en la nueva ruta de la seda.

\section{Conclusiones}

En primer lugar, cobra importancia la estabilidad geopolítica global y regional y la integración global de los mercados. Cabe preguntarse si hay una crisis 
global, no se sabe quién gobierna al mundo con una sensación de inseguridad y crisis global (Silva y Martins, 2013).

Se observa, a partir del gobierno de Trump, que Estados Unidos no ejerce el poder global de hace quince años; existen respuestas alternas como China, Rusia u otros con alcance como la Unión Europea, India, Japón, Korea. En este sentido, existe un poder más difuso, que hace que el control se vuelva compartido en más de un país, que proliferen los conflictos, los modelos económicos alternativos por guerras comerciales como la de los commodities, el acero, el petróleo (Rosas, 2008).

Entre los retos de Occidente se encuentra el de no tener forma de imponer políticas de manera unilateral. En este sentido, se observa cómo Alemania se repiensa si debe ser un poder geopolítico. China ha hecho una apuesta a través de la ruta de la seda para conquistar el mundo euroasiático, ya que no puede hacerlo Estados Unidos porque hace parte del continente americano (Gandasegui y Preciado, 2017).

América Latina en los últimos años ha experimentado un ingreso de presencia de potencias extrarregionales como China, Rusia, India, Turquía, Irán, que hace algunos años no lo hacían.

Finalmente, la dinámica de las TIC, las transformaciones económicas, la guerra, la cuarta revolución industrial, el surgimiento de nuevos poderes, nuevos desafíos a la gobernabilidad y un sentido más generalizado de inseguridad que incluye el terrorismo: resultantes del proceso de globalización (Murillo, 2015), serán características definitivas del mundo hasta 2020.

Así, a medida que mapeamos el futuro las perspectivas de aumentar la prosperidad global y la limitada la probabilidad de un gran conflicto de poder proporciona un ambiente general favorable para hacer frente a lo que de otra manera serían desafíos desalentadores (Berridge, 2010).

La presente investigación sirve como paso previo para estudios más consistentes a desarrollar en el futuro, que incluyan una investigación empírica encaminada a profundizar en el conocimiento de los escenarios posibles del orden mundial a 2020. Se debe reconocer que la presente investigación sufre todas las limitaciones de un trabajo esencialmente teórico y cualitativo. Por eso, se considera oportuno dar continuidad a este estudio planteando como futuras líneas de trabajo el desarrollo de una investigación empírica sobre los escenarios posibles del orden mundial en 2020 con acentuación en economía internacional que pueda aportar datos cuantitativos y estadísticamente relevantes. 


\section{Referencias}

Banco Asiático de Desarrollo, BAsD. (2011). Asia 2050. Realizing the Asian Century. Executive sumary. https://www.adb.org/sites/default/files/publication/28608/asia2050-executive-summary.pdf [Consultado el 2 de noviembre de 2018].

Barbieri, E. (1993). La previsión humana y Social. México D.F.: Fondo de Cultura Económica. Centro de Estudios Prospectivos.

Bascón, M.; Cazallo, A.; Lechuga, J.; Meñaca, I. (2016). Estudio de la necesidad de implantar un servicio público de transporte entre las ciudades de Ceuta-Tetuán y Melilla-Nador. Desarrollo Gerencial, 8(2), 37 - 57. http://revistas.unisimon.edu.co/index.php/desarrollogerencial/article/view/2553 [Consultado el 2 de noviembre de 2018].

Batta, V. (2013). Impulso a la enseñanza de la prospectiva en Relaciones Internacionales. Revista de Relaciones Internacionales de la UNAM, 116, 43-73. http://www.revistas.unam.mx/index.php/rri/article/view/49035 [Consultado el 2 de noviembre de 2018 ].

Bernal, C. (2010). Metodología de la investigación. Bogotá, Colombia: Pearson.

Berridge, G. (2010). Diplomacy: Theory and Practice. London, England: Palgrave.

Bustelo, P. (201 1). La UE ante el auge económico de Asia-Pacífico. Madrid, España: Real Instituto Elcano.

Carlsen, B.; Glenton, C. (2011). What About N? A Methodological Study of Sample-size Reporting in Focus Group Studies. BMC Medical Research Methodology, 11(26), 2-10. https://bmcmedresmethodol.biomedcentral. com/articles/10.1186/1471-2288-11-26 [Consultado el 2 de noviembre de 2018].

Creswell, J. W.; Miller, D. L. (2000). Determining validity in qualitative inquiry. Theory into Practice, 39(3), 124-130. https://www.tandfonline.com/doi/ abs/10.1207/s15430421tip3903_2 [Consultado el 2 de noviembre de 2018 ]. 
Davis, N. (2015). 4 scenarios for the future of civil society. https://www.weforum. org/agenda/2015/08/4-scenarios-for-the-future-of-civil-society/ [Consultado el 2 de noviembre de 2018 ].

Funabashi, Y.; Oksenberg, Y.; Weiss, H. (1994). China Una potencia emergente en un mundo de interdependencia (Informe para la Comisión Trilateral). Instituto INCIPE $N^{o}$ 6, 1-122. https://www.casaasia.es/ pdf/605041702321083855752986.pdf [Consultado el 2 de noviembre de 2018 ].

Gandasegui, M.; Preciado, J. (2017). Hegemonía y democracia en disputa. Trump y la geopolítica del conservadurismo. Guadalajara, México: Universidad de Guadalajara.

García-Hernández, G. E.; Manzano-Caudillo, J. (2010). Procedimientos metodológicos básicos y habilidades del investigador en el contexto de la teoría fundamentada. Iztapalapa. Revista de ciencias sociales y humanidades, 69, 1739. https://www.redalyc.org/articulo.oa?id=39348726002 [Consultado el 2 de noviembre de 2018 ].

González-Uresti, L. (2015) La Prospectiva: alcances y límites en Ciencias Sociales. Relaciones Internacionales, 48, 87 -108. https://revistas.unlp.edu.ar/ RRII-IRI/article/view/2201 [Consultado el 2 de noviembre de 2018 ].

Gorraiz, G. (2016). La economía mundial en el Trienio 2017-2020. https:// www.telesurtv.net/bloggers/La-economia-mundial-en-el-Trienio-2017-2020-20161217-0003.html\#comsup [Consultado el 2 de noviembre de 2018 ].

Hernández-Sampieri, R; Fernández, C; Baptista, P. (2014). Metodología de la investigación. México, D. F.: McGraw-Hill.

Hurtado, J. (2010). Guía para la comprensión holística de la ciencia. Caracas, Venezuela: Fundación Sypal.

Jouvenel, G. (1966). El Arte de la Conjetura. Madrid, España: Rialp

Lechuga, J. (2015). Prospectiva estratégica, vigilancia tecnológica e inteligencia competitiva: el caso del Instituto Tecnológico de Soledad Atlántico de Colombia. Revista Academia y Negocios, 1(2), 131-140. https://papers.ssrn. 
com/sol3/papers.cfm?abstract_id=2777538 [Consultado el 2 de noviembre de 2018].

Lechuga, J.; Cazallo, A.; Meñaca, I.; Martínez, H.; García, J. Olivero, E.; García, J. (2018). Análisis del comportamiento de las exportaciones del Departamento del Atlántico (Colombia) entre 2012-2016. Revista Espacios, 39(22), 2-20. http://www.revistaespacios.com/a 18v39n22/a18v39n22p22.pdf [Consultado el 2 de noviembre de 2018].

Manrique, C. (2016). La economía mundial en 2020. https://christianmanrique. com/2016/o1/18/la-economia-mundial-en-2020/

Martínez-Salgado, C. (2012). El muestreo en investigación cualitativa. Principios básicos y algunas controversias. Ciência E̊ Saúde Coletiva, 17(3), 613619. http://www.scielo.br/scielo.php?pid=S1413-81232012000300006\&s cript $=$ sci_abstract\&tlng $=$ es [Consultado el 2 de noviembre de 2018$]$.

Mendoza, R. (2006). Investigación cualitativa y cuantitativa. Diferencias y limitaciones. https://www.monografias.com/trabajos38/investigacion-cualitativa/investigacion-cualitativa2.shtml [Consultado el 2 de noviembre de 2018].

Mojica, F. (2010). Introducción a la prospectiva estratégica para la competitividad empresarial. Bogotá, Colombia: Cámara de Comercio de Bogotá.

Murillo, C. (2015). Diplomacia, inteligencia exterior y secreto diplomático frente a los desafíos de la era global. Revista Policía y Seguridad Pública, 5(2), 2974. https://www.lamjol.info/index.php/RPSP/article/view/2325 [Consultado el 2 de noviembre de 2018 ].

National Intelligence Council, NIC. (2004). Mapping the global future. Reporte del Proyecto del National Intelligence Council 2020. Pittsburg: Government Printing Office. https://www.dni.gov/files/documents/Global\%20 Trends_Mapping\%20the\%20Global\%20Future\%202020\%20Project.pdf [Consultado el 2 de noviembre de 2018].

Ohmae, K. (2005). The Next Global Stage: The Challenges and Opportunities in Our Borderless World. New Yersey, EE.UU: Prentice Hall 
Patiño, C. (2017). Imperios contra Estados: La destrucción del orden internacional contemporáneo. Bogotá, Colombia: Penguin Random House.

Paul, T. (2011). Las Potencias en ascenso y el equilibrio del poder en el siglo XXI. Revista Mexicana de Politica Exterior, 94, 95-1 16. https://revistadigital.sre.gob.mx/images/stories/numeros/n94/paul.pdf [Consultado el 2 de noviembre de 2018].

Pearson, F.; Rochester, J. M. (2000). Relaciones Internacionales. Situación Global en el Siglo XXI. México, D. F.: Ed. McGraw Hill.

Pérez, J. (2012). Cambio en el orden económico mundial. https://vaventura.com/ divulgacion/economia/cambio-orden-economico-mundial/ [Consultado el 2 de noviembre de 2018 ].

Rodríguez, J. M. (2017). Guerra comercial entre EE.UU. y China: ¿cuáles son las oportunidades para América Latina y el Caribe? [Video]. https://cnnespanol.cnn.com/video/eeuu-china-guerra-comercial-america-latina-caribe-economia-eduardo-oviedo-entrevista-rodriguez-dinero/ [Consultado el 2 de noviembre de 2018 ].

Rodríguez, K.; Gorjón, E. (2014). Grupos de Enfoque. En K. Saénz-López \& G. Tamez-González (Eds.). Métodos y técnicas cualitativas y cuantitativas aplicables a la investigación en ciencias sociales (pp. 148-170). México D. F.: Tirant Humanidades.

Rosas, C. (2008). China y Estados Unidos en el siglo XXI. Hacia una nueva bipolaridad. Comercio Exterior, 58(3), 198-217. http://revistas.bancomext. gob.mx/rce/magazines/113/3/RCE3.pdf [Consultado el 2 de noviembre de 2018].

Sandelowski, M.; Barroso, J. (2003). Writing the proposal for a qualitative research methodology project Qualitative. Health Research, 13(6), 781-820. https://www.ncbi.nlm.nih.gov/pubmed/12891715 [Consultado el 2 de noviembre de 2018]. 
Schiavon, J.; Ortega, A.; López-Vallejo, M.; Velázquez, R. (2014). Teorías de las Relaciones Internacionales en el siglo XXI: Interpretaciones críticas desde México. México, D. F: BUAP y UPAEP.

Schwartz, P. (1997). La planificación estratégica por escenarios. Cuadernos de Administración, 21, 200-225.http://revistas.univalle.edu.co/index.php/cuadernos_de_administracion/article/view/114 [Consultado el 2 de noviembre de 2018].

Silva, C.; Martins, C. (2013). Nuevos escenarios para la integración en América Latina. http://biblioteca.clacso.edu.ar/clacso/gt/20131016025228/NuevosEscenarios.pdf [Consultado el 2 de noviembre de 2018].

Strauss, A.; Corbin, J. (1990). Basics of qualitative research. Thousand Oaks, California: Sage.

Taylor, S. J.; Bogdan, R. (1986). Introducción a los métodos cualitativos de investigación. México, D. F.: Paidós.

The Asian Development Bank, ADB (2011). Asia 2050: Realizing the Asian Century. Singapur, China: Asian Development Bank.

Trejos, R. (1997). Hacia un desarrollo sostenible de la agricultura y el medio rural. San José, Costa Rica: Inter-American Institute for Cooperation on Agriculture, IICA.

Zeraoui, Z.; Sarquis, D.; González, L.; Perni Orietta, T. P.; Balderrama, R. (2014). Los procesos Mundiales Contemporáneos: Un Mundo Transformado. México, D. F: Trillas. 
